\title{
Estudio dialectológico perceptual de las Antillas hispánicas
}

\author{
Roxana Sobrino Triana (Universidad de Bergen, Noruega)
}

\begin{abstract}
In this paper I analyzed the Spanish - speaking Caribbean's dialectal perceptions (Cubans, Puerto Ricans and Dominicans), in order to know the mental delimitation of the linguistic area to which their varieties are circumscribed, the elements through which they distinguish it and the way in which they perceived themselves linguistically in the Spanish-speaking context in general. The analysis has been based on the postulates of Perceptual Dialectology. From the methodological point of view, a questionnaire was applied in the capitals of the three countries in which four questions addressed the linguistic perceptions. The sample was formed from a fixed quota sampling, for a total of 198 informants surveyed in the three countries, of which the variables sex, age and level of education were controlled.

Some of the most important concluding ideas of the study are that the Antillean perceive their varieties of Spanish as inserted in a common dialectal space, although with variable limits. In a triangle of relationships, Puerto Ricans and Cubans perceive their respective varieties of Spanish as much closer and more like each other than to the Spanish in the Dominican Republic. Dominicans, on the other hand, show a weaker awareness of the link between the three varieties. As for the differentiating perceptions, is evident a perceptual distance from other variants (such as the Spanish, Colombian, Mexican and Argentine), based on arguments that show the prestige granted to these modalities of language.
\end{abstract}

Keywords: linguistic perceptions, Spanish-speaking Antilles, Perceptual Dialectology, mental maps, perceptual dialectal zone

\section{Introducción}

El modo en que los hablantes perciben sus lenguas, así como las creencias que subyacen en el proceso de percepción de la realidad lingüística, han devenido objeto de estudio de la dialectología perceptual como disciplina. Si bien los trabajos dialectológicos perceptuales sobre lenguas como el inglés, el alemán o el japonés, han tenido fuerza en los últimos años (Preston 1999a; Long y Preston 2002), sobre el español el interés es notablemente reciente y se contabilizan solo algunos pocos. De ellos, una parte se ha enfocado en la percepción hacia las variedades hispánicas de manera global (Quesada 2014; Moreno 2015) y otros se acercan al tema analizando las variedades nacionales. Esta última línea de investigación ha sido hasta el momento la más desarrollada y se localizan investigaciones relacionadas con las variedades de España (Moreno y Moreno 2002), México (Serrano 2002; Erdösova 2011), Cuba (Sobrino 2013), Costa Rica (Quesada 2013) y Venezuela (Castro y Malaver 2016). 
Asimismo, otros estudios vinculan resultados de la dialectología perceptual y las actitudes lingüísticas. Es el caso de Erdösova (2011) para el español de México, Bravo (2015) para las variedades murcianas y el proyecto "Identidad y actitudes lingüísticas hacia el español" (LIAS) (Chiquito y Quesada 2014). Sobre la vinculación de ambas disciplinas se ha apuntado, en forma de reclamo, el hecho de que los estudios sobre actitudes lingüísticas no relacionan sus resultados a los obtenidos por la dialectología perceptual, ${ }^{1}$ y es que indagar sobre la percepción dialectal de los hablantes en una investigación sobre actitudes lingüísticas es profundizar en uno de los componentes que las motivan.

Precisamente, los resultados que se presentan en este artículo forman parte de una tesis doctoral sobre actitudes lingüísticas en el Caribe insular hispánico (Sobrino 2017), la cual se incluye en este pequeño grupo de estudios sobre variedades de español que relacionan los postulados de ambas disciplinas. En este trabajo se abordarán, específicamente, las percepciones dialectales de los hablantes de las Antillas (Cuba, Puerto Rico y República Dominicana) con el objetivo de identificar las similitudes y diferencias que perciben entre sus propias variedades y el resto de las variedades hispánicas, la configuración mental que hacen del área lingüística en la que se incluyen, los elementos a través de los cuales la distinguen y los límites a los que la circunscriben.

\section{Marco teórico}

La dialectología perceptual (DP) se considera una subrama de la lingüística popular (folk linguistics) y se encarga de estudiar las percepciones de los hablantes comunes sobre la lengua. Sus presupuestos básicos se fundamentan en la existencia de una Teoría popular de la lengua (Preston 2004) según la cual, en las creencias populares, la lengua se concibe como algo auténtico y palpable, como una realidad extra cognitiva a la que los hablantes se adscriben de mejor o peor manera.

La DP se propone trazar un cartografiado lingüístico, a semejanza de la dialectología tradicional, pero con base en la percepción dialectal de los hablantes, en las creencias que almacenan en su conciencia lingüística. A diferencia de la geografía lingüística que construye sus mapas a través del análisis de datos lingüísticos recogidos en el terreno, en este caso se trata de mapas mentales construidos por los hablantes comunes en los que trazan límites o isoglosas según sus percepciones y la construcción de estereotipos.

La percepción lingüística y la consecuente interpretación de esas percepciones que el hablante experimenta, manifiestas a través de opiniones, son concebidas del siguiente modo:

\footnotetext{
"La percepción lingüística es básicamente la capacidad de recibir impresiones o sensaciones procedentes del uso de las lenguas. Por su parte, la opinión lingüística puede definirse como la interpretación que los hablantes hacen del posicionamiento social y geográfico de las lenguas. Percepción y opinión son, pues, conceptos traslapados en los que tan importante es el acceso a la realidad lingüística y su consecuente percepción física, como el procesamiento cognitivo e intelectual que los hablantes hacen de las sensaciones experimentadas (Moreno 2015, 217).
}

\footnotetext{
${ }^{1}$ Según Preston (1999b, 129), "los estudios 'clásicos' sobre actitudes lingüísticas no prestan atención a la conciencia lingüística popular de los encuestados (ni a las estrategias de identificación que utilizan para 'localizar' a los hablantes ni a los 'mapas mentales' que tienen sobre las áreas de habla regional).
} 
Dado que el propósito último de la DP es la representación en mapas de las percepciones de los hablantes, la metodología empleada con más frecuencia parte de presentar al informante un mapa del área de interés, generalmente en blanco, para que el hablante represente en él las diferentes zonas que percibe según la manera de hablar. Además de estas, se han empleado otras técnicas tales como escalas de diferenciación (para establecer el grado de percepción en cuanto a la diferencia de otros dialectos con el propio), identificación de dialectos (a través de grabaciones de variedades dialectales que el informante debe identificar y ubicar en un mapa) y datos cualitativos (entrevistas y preguntas abiertas) (Preston 1999a, xxxiv).

Las investigaciones en este campo han enunciado algunos principios de gran utilidad para analizar las percepciones que manifiestan los hablantes sobre las variedades dialectales. Uno de ellos, el «principio o efecto de proximidad» (Montgomery 2012, 640), plantea que los hablantes tienden a considerar las áreas cercanas con mayor facilidad que otras áreas. No obstante, la proximidad no debe ser entendida solamente como espacio geográfico. Se da el caso de que los hablantes toman en consideración variedades de lengua geográficamente distantes a la suya y aquí entran en juego factores sociales que incrementan el efecto de proximidad, tales como los medios de difusión y las redes sociales. Asimismo, el impacto de la proximidad puede ser modificado por la relativa importancia cultural de un área, ya sea estigmatizada o prestigiosa. El efecto de la importancia cultural incrementará la proximidad de un área a los informantes y, por tanto, aumentará las posibilidades de esa área de ser incluida en sus mapas mentales (Ibídem, 662).

Otro de los principios tomados en consideración es el relacionado con la idea de un «continuum dialectal perceptual», según el cual A se parece a $\mathrm{B}, \mathrm{B}$ se parece a $\mathrm{C}$ y $\mathrm{C}$ a D, pero A no se parece a D (Quesada 2014, 292); lo que se entendería como que, a mayor distancia geográfica mayor diferenciación perceptual, en sintonía también con el principio de proximidad.

Por su parte, el «principio o eje de la reciprocidad» se compone de dos parámetros: el «parámetro de la reciprocidad»y «el de la no reciprocidad» ${ }^{2}$. En el «eje de la reciprocidad»se ubican aquellos casos en los que un hablante de un país A ve similitudes con los de un país B y viceversa. El «parámetro de la no reciprocidad» consistiría en que A percibe similitudes con $\mathrm{B}$, pero B no con A. Tal ruptura de la reciprocidad se explica con la posible influencia de factores sociales e históricos, donde puede desempeñar un papel importante el prestigio lingüístico. Es decir, un país con una variedad de lengua no prestigiosa (A) puede considerarse similar a otro (B) basado en el prestigio del que este puede gozar; sin embargo, el prestigioso (B), no considerará semejante a este otro (A), al no gozar de igual prestigio lingüístico.

\section{Metodología}

Para la obtención de los datos se empleó un cuestionario, aplicado en el año 2015 en cada una de las capitales de los países estudiados: La Habana, Cuba; San Juan, Puerto Rico y Santo Domingo, República Dominicana. La muestra se conformó a partir de un muestreo por estratificación uniforme tomando en consideración las variables sociolinguiísticas sexo, edad y nivel de instrucción, para un total de 198 informantes.

${ }^{2}$ Los principios del continuum dialectal, el de la reciprocidad y no reciprocidad, fueron enunciados por Quesada (2014) a partir del análisis dialectológico perceptual aplicado a hablantes de español en un estudio panhispánico. 
Se trabajó con tres grupos etarios con edades comprendidas entre los 20 y 34 años (IGE), 35 a 54 años (IIGE) y mayores de 55 (IIIGE). Los niveles de instrucción tomados en consideración se ajustaron a nivel bajo (NB) para informantes con un máximo de noveno grado, nivel medio (NM) para aquellos con un máximo de educación técnica o duodécimo grado y nivel alto (NA) para los informantes con estudios universitarios completados.

La muestra quedó conformada del modo siguiente (Tabla 1):

\begin{tabular}{|l|ccc|ccc|} 
& \multicolumn{3}{|c|}{ Sexo femenino } & \multicolumn{3}{c|}{ Sexo masculino } \\
$\begin{array}{l}\text { Grupos } \\
\text { etarios }\end{array}$ & NB & NM & NA & NB & NM & NA \\
\hline IGE & $4 / 3^{3}$ & $4 / 3$ & $4 / 3$ & $4 / 3$ & $4 / 3$ & $4 / 3$ \\
\hline IIGE & $4 / 3$ & $4 / 3$ & $4 / 3$ & $4 / 3$ & $4 / 3$ & $4 / 3$ \\
\hline IIIGE & $4 / 3$ & $4 / 3$ & $4 / 3$ & $4 / 3$ & $4 / 3$ & $4 / 3$ \\
\hline $\begin{array}{l}\text { Total de } \\
\text { informantes }\end{array}$ & & \multicolumn{5}{c}{$72 / 72 / 54$} \\
\end{tabular}

Tabla 1: Número de informantes por variables sociolingüísticas tomadas en consideración siguientes:

Las preguntas sobre percepción dialectal incluidas en el cuestionario fueron las

1. Mencione otros países donde hablan el español de modo similar a como usted lo habla

2. ¿En qué consisten esas semejanzas?

3. Mencione otros países donde hablan el español de modo diferente a como usted lo habla

4. ¿En qué consisten esas diferencias?

Las respuestas ofrecidas a las preguntas 1 y 3 han sido representadas en mapas por países y se han elaborado mapas generales de semejanzas y diferencias, con el propósito fundamental de visualizar el área dialectal que los antillanos conciben en sus mapas mentales. Por su parte, las respuestas a las preguntas 2 y 4 (argumentos de semejanzas o diferencias), al tratarse de preguntas abiertas, fueron codificadas a posteriori y se sistematizaron en criterios que facilitaran el análisis.

\section{Presentación de resultados}

\subsection{Percepción de semejanzas}

La percepción de semejanzas entre la variedad propia y otras del español fue similar en los tres países estudiados, pero algunos elementos distinguen el modo en que se visualizan

\footnotetext{
${ }^{3}$ El 4 representa el número de informantes por celda en Cuba y República Dominicana y el 3 en Puerto Rico. La diferencia se debe a que el Área Metropolitana de Puerto Rico apenas cuenta con una población de 952970 , mientras que el número de habitantes de La Habana asciende a 2125320 y a 3339410 en Santo Domingo.
} 
cubanos, puertorriqueños y dominicanos insertos en una zona común. Las variedades consideradas como semejantes a la propia por los cubanos son las de Puerto Rico, República Dominicana y Venezuela, seguidas de Panamá y Colombia; el resto de las variantes mencionadas posee porcentajes muy bajos (Mapa 1). En el caso de los puertorriqueños, ellos conciben un espacio hispanohablante con similitudes algo mayor que los cubanos, donde los vínculos con Colombia y Panamá parecen ser algo más estrechos (Mapa 2). Los dominicanos, a diferencia de sus vecinos, rompen la trinidad antillana que se manifiesta en los mapas correspondientes a Cuba y Puerto Rico. En este caso, los puertorriqueños son los que más se mencionan como semejantes pero, a continuación, en orden de frecuencia de mención, aparecen Venezuela, México y Cuba, estos dos últimos con iguales porcentajes. Igualmente, España es más mencionada que Colombia y Panamá (Mapa 3). Todo ello indicaría que los dominicanos poseen una visión mucho más amplia del área dialectal a la que pertenecen.

Aunque las unidades de percepción pensadas para el análisis se ajustaban a países; es decir, a territorios nacionales hispanohablantes, en algunos casos se hizo referencia a una región de España (Islas Canarias) y en otra se tomó en cuenta el área caribeña ("los del Caribe"). Algo similar sucede con Estados Unidos: puertorriqueños y dominicanos consideran ciudades como Orlando, Nueva York, Miami y el estado de la Florida, donde radican importantes comunidades de estos países.

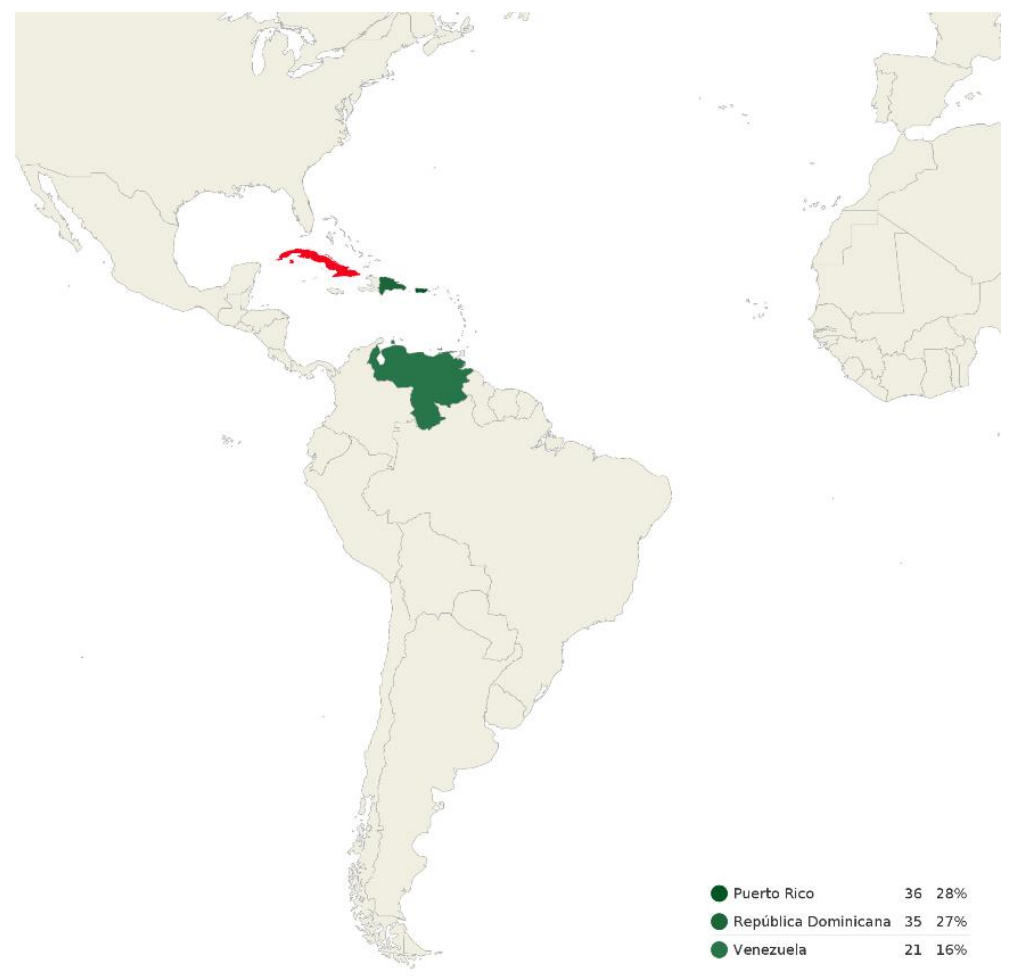

Mapa 1: Lugares percibidos como semejantes por los cubanos ${ }^{4}$

\footnotetext{
${ }^{4}$ En los mapas que ilustran las percepciones de los informantes en cuanto a semejanzas por cada país (Mapas 1, 2 y 3), el país objeto de estudio aparece representado en color rojo, mientras que las respuestas ofrecidas están en color verde. Solo se han representado aquellos lugares que fueron mencionados con frecuencias por encima de la media.
} 


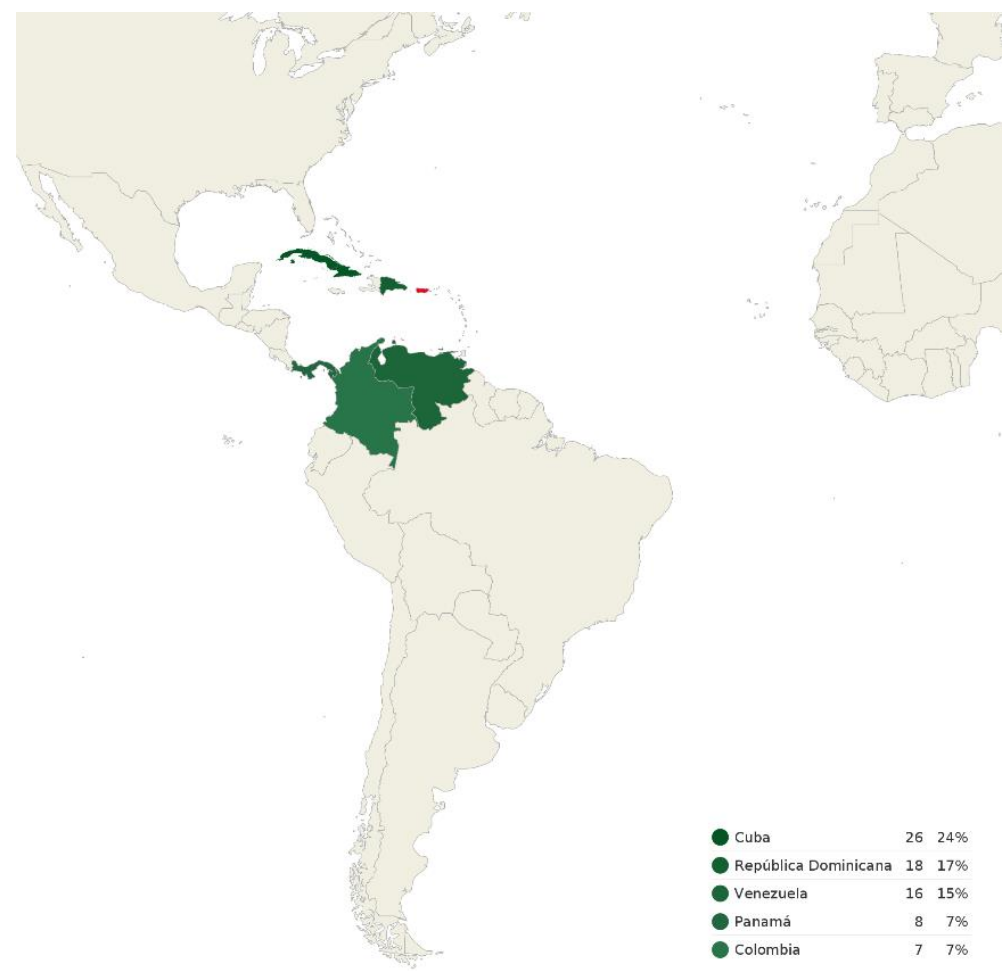

Mapa 2: Lugares percibidos como semejantes por los puertorriqueños

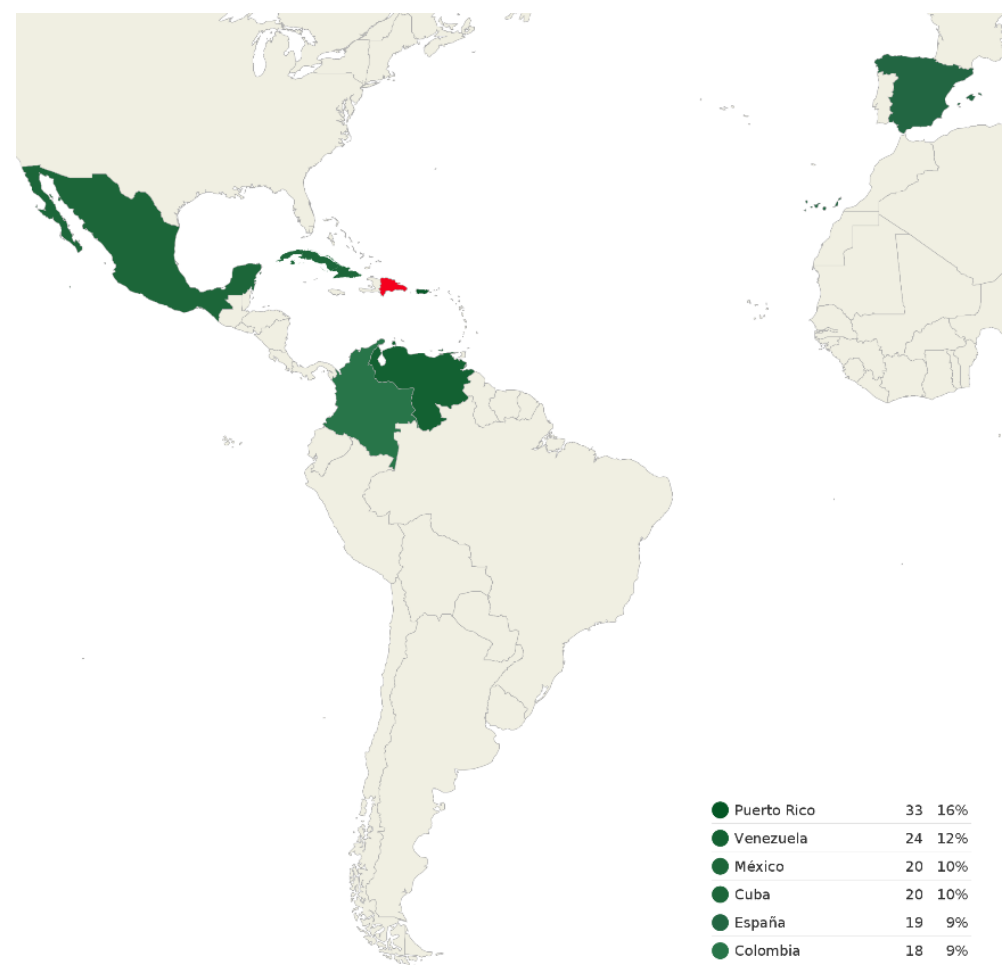

Mapa 3: Lugares percibidos como semejantes por los dominicanos

Las respuestas ofrecidas por los informantes se clasificaron según el número de variedades de lengua mencionadas. En cubanos y puertorriqueños predominaron las 
respuestas que se han dado en llamar monoperceptuales (33,3\% en ambos países) y biperceptuales (29,3\% los cubanos y 33,2 \% los puertorriqueños), en las que se consideró similar a su modo de hablar la variedad de uno o dos países, respectivamente, los cuales coinciden en casi todos los casos con sus vecinos antillanos. Por el contrario, las respuestas más frecuentes de los dominicanos son las que contienen de dos a cuatro lugares $(70,8 \%)$; es decir, los mapas mentales que construyen los dominicanos están integrados, con más frecuencia, por un mayor número de variedades. En solo 12,5\% de los casos los dominicanos ofrecieron respuestas monoperceptuales en las que vinculan su variedad con un solo lugar que, generalmente, es España o Venezuela, no una de las Antillas.

Según se ilustra en la Tabla 2, los criterios en los que los antillanos basan sus percepciones de similitudes entre variedades son fundamentalmente criterios lingüísticos, sobre todo en los niveles léxico y fónico, así como el nivel suprasegmental. Menos frecuentes son aquellos argumentos que toman en cuenta criterios de orden extralingüístico, como pueden ser cuestiones de comunidad cultural, identidad, cercanía geográfica, entre otros.

\begin{tabular}{|c|c|c|c|c|c|c|c|}
\hline \multicolumn{2}{|c|}{$\begin{array}{l}\text { Criterios para el } \\
\text { establecimiento de semejanzas } \\
\text { lingüísticas }^{5}\end{array}$} & \multicolumn{2}{|l|}{$\mathbf{C u}$} & \multicolumn{2}{|l|}{$\mathbf{P R}$} & $\begin{array}{r}\text { RD } \\
\text { Menciones }\end{array}$ & $\%$ \\
\hline \multirow{12}{*}{ 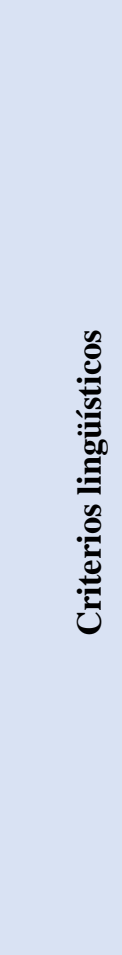 } & nivel léxico & 11 & 12,9 & 21 & 27,6 & 10 & 13,5 \\
\hline & $\begin{array}{l}\text { nivel } \\
\text { suprasegmental }\end{array}$ & 14 & 16,5 & 12 & 15,8 & 7 & 9,5 \\
\hline & nivel fónico & 7 & 8,2 & 9 & 11,8 & 1 & 1,4 \\
\hline & lengua común & - & - & 2 & 2,6 & 32 & 43,2 \\
\hline & espanglish & - & - & 1 & 1,3 & - & - \\
\hline & nivel sintáctico & 1 & 1,2 & 1 & 1,3 & - & - \\
\hline & $\begin{array}{l}\text { semejanza con el } \\
\text { oriente de Cuba }\end{array}$ & 16 & 18,8 & - & - & - & - \\
\hline & forma de hablar & 6 & 7,1 & 4 & 5,3 & 1 & 1,4 \\
\hline & incorrección & 1 & 1,2 & 1 & 1,3 & - & - \\
\hline & comprensión & 1 & 1,2 & 3 & 3,9 & 2 & 2,7 \\
\hline & rapidez elocutiva & - & - & 2 & 2,6 & 1 & 1,4 \\
\hline & Subtotal & 57 & 67,1 & 56 & 73,7 & 54 & $\mathbf{7 3 , 0}$ \\
\hline
\end{tabular}

\footnotetext{
${ }^{5}$ Las respuestas aportadas para argumentar las semejanzas y diferencias entre las variedades mencionadas (Tablas 2 y 3) fueron agrupadas en diferentes criterios. Aquellas respuestas relacionadas con los diferentes niveles lingüísticos se agruparon en los criterios denominados nivel fónico, léxico, sintáctico y suprasegmental. El resto de los criterios lingǘsticos se refieren a: la idea de la fuerte similitud entre la parte oriental de Cuba y las Antillas (semejanza con el oriente de Cuba), facilidad de comprensión (comprensión), criterios generalizadores sobre las similitudes que no especifican ningún aspecto (forma de hablar), semejanzas en cuanto a "usos incorrectos" (incorrección). Por su parte, los criterios extralingüísticos reúnen las respuestas relacionadas con las semejanzas en cuanto a rasgos culturales, históricos e identitarios (cultura, historia, identidad), a las similitudes de los orígenes étnicos de los grupos conformadores de estas naciones (origen étnico) y la proximidad geográfica (cercanía geográfica).
} 


\begin{tabular}{|c|c|c|c|c|c|c|c|}
\hline \multirow{6}{*}{ 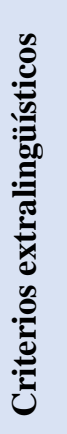 } & cultura & 6 & 7,1 & 8 & 10,5 & 4 & 5,4 \\
\hline & identidad & 5 & 5,9 & 1 & 1,3 & - & - \\
\hline & origen étnico & 1 & 1,2 & - & - & - & - \\
\hline & cercanía geográfica & 1 & 1,2 & 1 & 1,3 & 1 & 1,4 \\
\hline & historia & - & - & - & - & 1 & 1,4 \\
\hline & Subtotal & 13 & 15,3 & 10 & 13,2 & 6 & 8,1 \\
\hline \multirow{5}{*}{$\stackrel{\mathscr{E}}{0}$} & no responde & 6 & 7,1 & 2 & 2,6 & 7 & 9,5 \\
\hline & no sé & 2 & 2,4 & 5 & 6,6 & 7 & 9,5 \\
\hline & otra & 7 & 8,2 & 3 & 3,9 & - & - \\
\hline & Subtotal & 15 & 17,6 & 10 & 13,2 & 14 & 18,9 \\
\hline & Total de menciones & 85 & 100 & 76 & 100 & 74 & 100 \\
\hline
\end{tabular}

Tabla 2: Criterios para el establecimiento de semejanzas

Los cubanos se distinguen por destacar las similitudes de los vecinos con la zona oriental del país $(18,8 \%)$. Los de Puerto Rico son los que más atienden a elementos del vocabulario (27,6\% de las menciones se ubican en este criterio), ya sea el significado de las palabras, expresiones y voces propias. Asimismo, son los únicos en tomar en cuenta el uso del espanglish como patrón de semejanza. Los dominicanos, por su parte, toman en cuenta menos criterios que sus vecinos; casi la mitad de las ideas aportadas $(43,2 \%)$ se corresponden con el hecho de que comparten la misma lengua, son ellos los que más dudan $(9,5 \%)$ y los que más evitan responder a esta pregunta $(9,5 \%)$. fueron:

Algunos de los argumentos para justificar las similitudes percibidas entre variedades

- Cuba sobre Puerto Rico: los puertorriqueños se parecen a los orientales (Santiago de Cuba, Guantánamo $\left.{ }^{6}\right)$, en la pronunciación se asemejan un poco, en la dicción, las expresiones, la forma de ser, lo jaraneros, cariñosos, se dan fácil a querer;

- Puerto Rico sobre República Dominicana: la semejanza es que no hablamos el español correctamente, cometemos muchos errores, por ejemplo, cambiamos la erre por la ele;

- Puerto Rico sobre Cuba y República Dominicana: el español es similar, lo que cambia es el acento o palabras con diferente significado;

- República Dominicana sobre Paraguay, Venezuela, Puerto Rico, Cuba: todos hablamos el mismo castellano.

\footnotetext{
${ }^{6}$ Santiago de Cuba y Guantánamo son dos provincias de la región oriental de Cuba.
} 


\subsection{Percepción de diferencias}

Entre las variedades marcadamente diferenciadas de la propia, los cubanos consideran con más frecuencia la argentina, mexicana y española. En general, se hace mención de casi todas las variedades nacionales, incluidas algunas que coinciden con las más mencionadas por los antillanos cuando se trataba de las semejanzas, como el español de Colombia y Venezuela, por ejemplo (Mapa 4). Los puertorriqueños, por su parte, coincidieron en los lugares más mencionados con los cubanos, aunque es de destacar que la mención de República Dominicana fue más frecuente $(5 \%)$ que la de otros países lejanos geográficamente. También se refirieron a regiones españolas, como Cataluña y el País Vasco, y es notable el área que se extiende desde Colombia hasta Argentina, reconocida casi en su totalidad como marcadamente diferente (Mapa 5).

Si bien de los sitios más mencionados por los dominicanos la mayoría coincide con sus vecinos antillanos, la mención del habla puertorriqueña como segunda variedad más frecuente $(17 \%)$ es un dato que aporta valiosa información sobre los vínculos particulares entre estas dos variantes, según sus hablantes. Asimismo, Cuba, Colombia y Venezuela poseen frecuencias significativas (6\% para cada uno de estos países). Destaca también la referencia a la ciudad de Nueva York (4\%), posiblemente relacionada con la numerosa población de origen puertorriqueño que allí radica (Mapa 6).

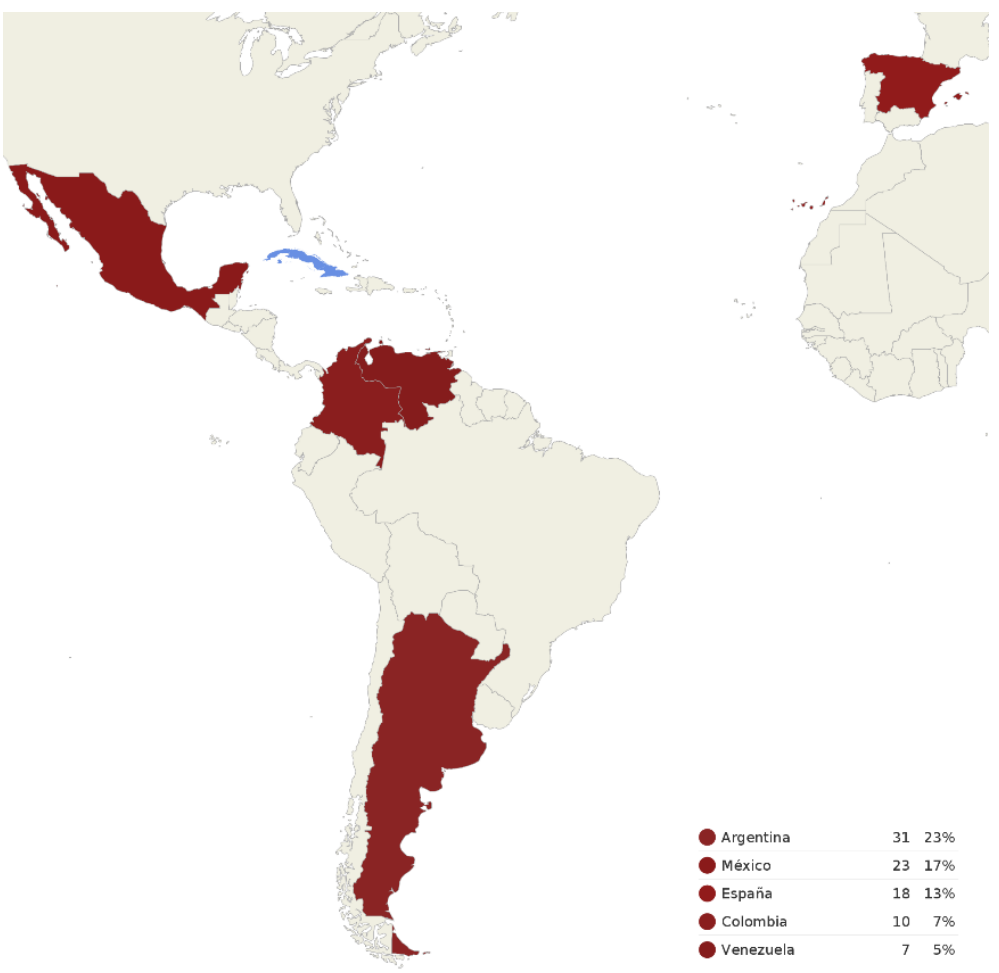

Mapa 4: Lugares percibidos como diferentes por los cubanos ${ }^{7}$

\footnotetext{
${ }^{7}$ En los mapas que se refieren a las variedades como diferentes (Mapas 4, 5 y 6), el lugar de donde proviene la información se representa con color azul y la representación de los sitios mencionados está en color rojo. De modo similar a los anteriores, solo se han representado los lugares que tuvieron una frecuencia de mención mayor al promedio.
} 


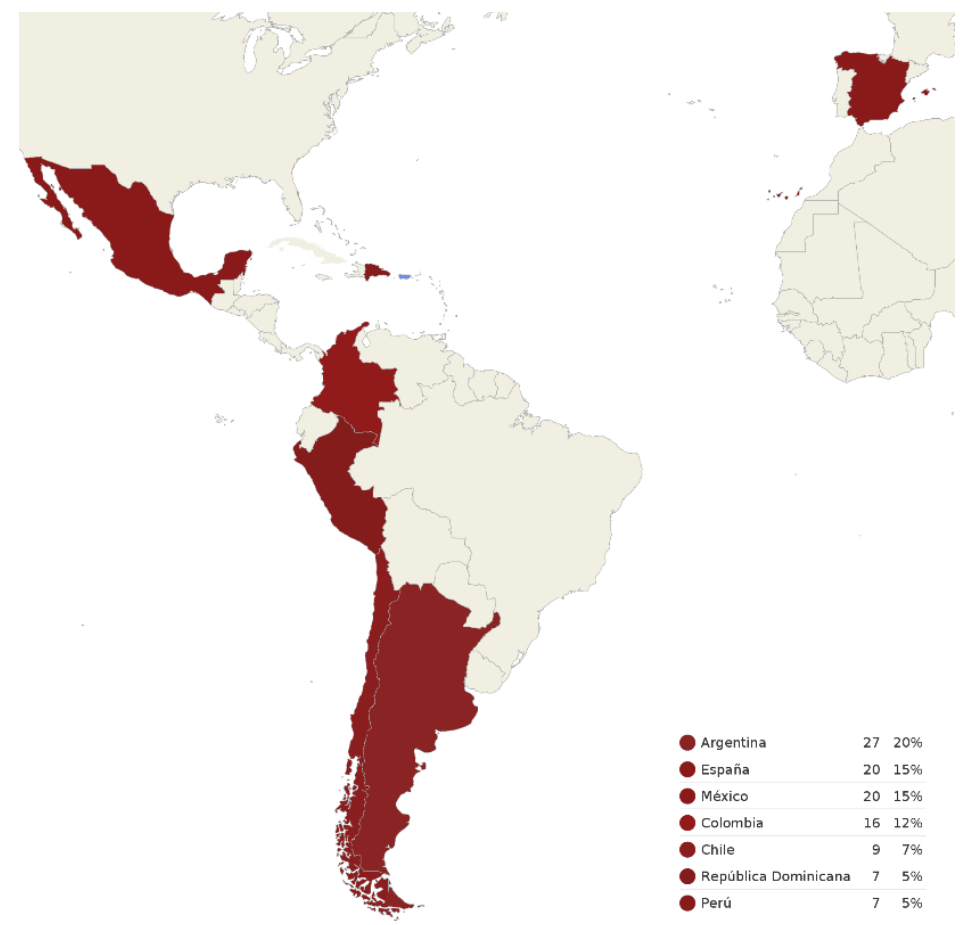

Mapa 5: Lugares percibidos como diferentes por los puertorriqueños

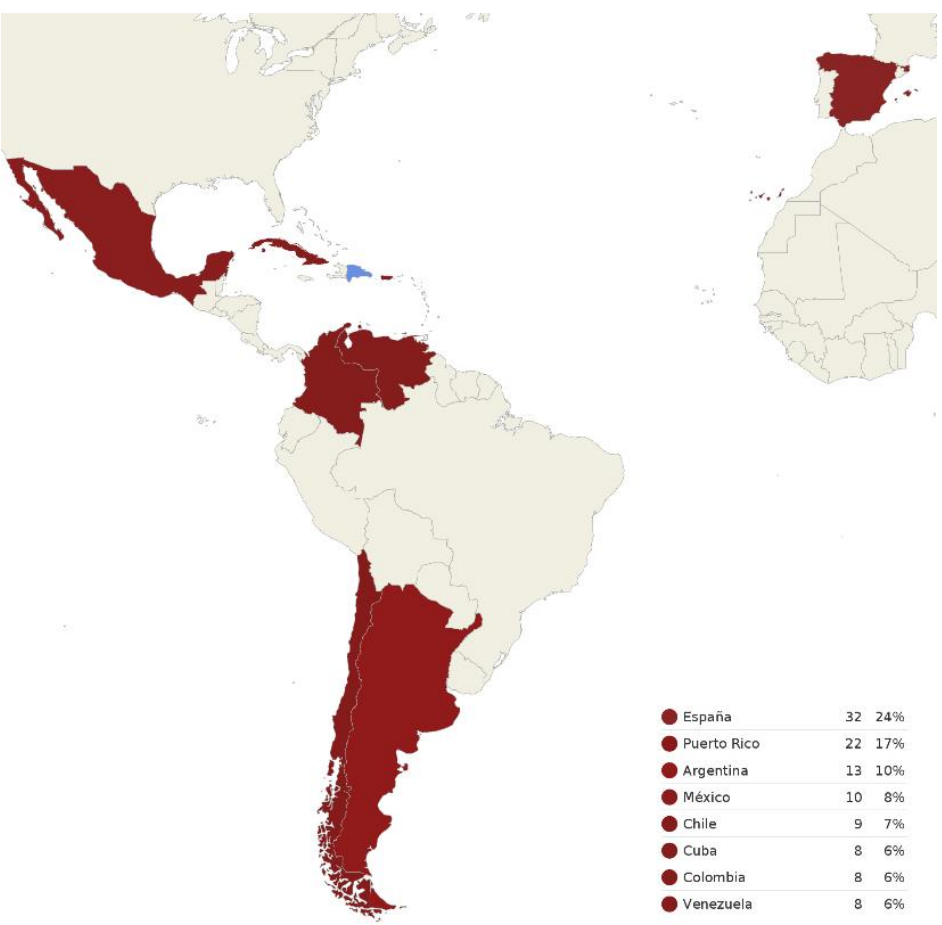

Mapa 6: Lugares percibidos como diferentes por los dominicanos

En cuanto a los criterios en los que basan tales percepciones, los antillanos toman en consideración, fundamentalmente, aspectos lingüísticos, de modo similar a lo sucedido con las percepciones de variantes semejantes (Tabla 3). Sin embargo, en este caso el predominio de los criterios de orden lingüístico sobre el resto es aún mayor, por encima del $80 \%$ en los 
tres países. En consecuencia, las referencias a cuestiones extralingüísticas son también menores, sobre todo en República Dominicana, donde apenas alcanzan un 2,8\%. Aquellas respuestas agrupadas en "Otros criterios" solo son significativas en el caso dominicano $(15,0 \%)$, en los otros dos países los informantes apenas dejan de responder o dicen no saber. Todo ello puede llevar a la idea de que, al menos cubanos y puertorriqueños, tienen mayor claridad de aquellos elementos lingüísticos que distinguen a sus respectivas variantes de lengua que de aquellos que los asemejan, por esto describen más rasgos lingüísticos diferenciales y recurren más a cuestiones culturales y extralingüísticas para establecer semejanzas.

\begin{tabular}{|c|c|c|c|c|c|c|c|}
\hline \multirow{2}{*}{\multicolumn{2}{|c|}{$\begin{array}{l}\text { Criterios para el establecimiento de } \\
\text { diferencias lingüísticas }\end{array}$}} & \multicolumn{2}{|l|}{$\mathbf{C u}$} & \multicolumn{2}{|l|}{ PR } & \multicolumn{2}{|c|}{ RD } \\
\hline & & Menciones & $\%$ & Menciones & $\%$ & Menciones & $\%$ \\
\hline \multirow{12}{*}{ 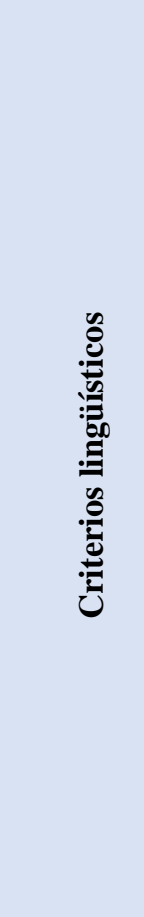 } & nivel léxico & 19 & 17,8 & 20 & 26,0 & 18 & 16,8 \\
\hline & nivel suprasegmental & 36 & 33,6 & 29 & 37,7 & 34 & 31,8 \\
\hline & nivel fónico & 20 & 18,7 & 10 & 13,0 & 24 & 22,4 \\
\hline & nivel sintáctico & 1 & 1,0 & - & - & - & - \\
\hline & influencia de otras lenguas & - & - & 3 & 3,9 & 6 & 5,6 \\
\hline & formas de tratamiento & 7 & 6,5 & 2 & 2,6 & - & - \\
\hline & $\begin{array}{l}\text { semejanza con el oriente } \\
\text { de Cuba }\end{array}$ & 3 & 2,8 & - & - & - & - \\
\hline & forma de hablar & 4 & 3,7 & 1 & 1,3 & 5 & 4,7 \\
\hline & corrección & 1 & 1,0 & 2 & 2,6 & - & - \\
\hline & incomprensión & 1 & 1,0 & - & - & 1 & 1,0 \\
\hline & rapidez elocutiva & 2 & 1,9 & 1 & 1,3 & - & - \\
\hline & Subtotales & 94 & 87,9 & 68 & 88,3 & 88 & 82,2 \\
\hline \multirow{5}{*}{ } & cultura & 5 & 4,7 & 1 & 1,3 & 3 & 2,8 \\
\hline & origen étnico & 3 & 2,8 & 1 & 1,3 & - & - \\
\hline & ubicación geográfica & - & - & 1 & 1,3 & - & - \\
\hline & migraciones & - & - & 2 & 2,6 & - & - \\
\hline & Subtotales & 8 & 7,5 & 5 & 6,5 & 3 & 2,8 \\
\hline \multirow{5}{*}{$\stackrel{\mathscr{0}}{0}$} & no responde & 2 & 1,9 & 1 & 1,3 & - & - \\
\hline & no sé & 1 & 1,0 & 1 & 1,3 & 12 & 11,2 \\
\hline & otra & 2 & 1,9 & 2 & 2,6 & 4 & 3,7 \\
\hline & Subtotales & 5 & 4,7 & 4 & 5,2 & 16 & 15,0 \\
\hline & Total de menciones & 107 & 100 & 77 & 100 & 107 & 100 \\
\hline
\end{tabular}

Tabla 3: Criterios para el establecimiento de diferencias 
En los tres países las ideas relacionadas con las diferencias suprasegmentales (acentuales y entonativas) fueron las más frecuentes, seguidas de las referencias a la diversidad en el vocabulario y la pronunciación. Los cubanos son los únicos en recurrir a aspectos gramaticales y en tomar como referencia el oriente del país. Las semejanzas con el oriente cubano fue el criterio más mencionado en Cuba cuando se trataba de similitudes y está presente también cuando se trata de establecer diferencias, aunque es menos frecuente. Este mecanismo de relación de acercar lo menos conocido a partir de su vinculación con una realidad más cercana no fue privativo de los cubanos, también los puertorriqueños lo hicieron al establecer diferencias entre la variedad dominicana y la propia a través de la relación de estos con la forma de hablar de Lares, e igualmente los capitalinos dominicanos al vincular la variedad puertorriqueña con la región de El Cibao ${ }^{8}$.

En los tres casos se asocian las variedades vecinas con una modalidad de habla del país que es estigmatizada, de modo que se discrimina indirectamente la variante en cuestión. Estudios cubanos han confirmado la estigmatización de que es víctima el habla de la región oriental, sobre todo por parte de los capitalinos (Fúster 2012, 145; Sobrino et al. 2014, 324325). Por tanto, el relacionar las variedades dominicana, puertorriqueña y venezolana con el oriente del país es un modo de discriminación, al tiempo que constituye un recurso del hablante habanero de establecer distancia y jerarquía entre su modo de hablar y el resto de los que integran el área.

La similitud del oriente cubano con el resto de las Antillas hispanohablantes es un punto en el que han coincidido lingüistas y no lingüistas, y son diversas las causas supuestas. Algunas de ellas se relacionan con el hecho de que los lazos lingüísticos y comerciales del oriente fueron más estrechos en la etapa colonial con otras islas del Caribe que con La Habana (Lipski 2004: 252); las migraciones internas (Valdés 1999) y, en general, coincidencias sociohistóricas de causación múltiple (Choy 1999).

Algunos ejemplos de los argumentos expuestos para justificar las percepciones de diferencias son:

- Cuba sobre Argentina: usan palabras distintas y no pronuncian igual; en lugar de decir tú, dicen vos; en la pronunciación, hablan con la ye, che; dicen che; los argentinos tienen diferente entonación; habla bien el español, bien pronunciado;

- Cuba sobre España: por la entonación y las palabras que usan: de puta madre; usan la zeta, ellos tienen más cultura; la pronunciación de ellos es más exacta;

- Cuba sobre Colombia: los colombianos le dicen usted a todo el mundo;

- Cuba sobre República Dominicana: hablan feo, son medio analfabetos;

- Puerto Rico sobre República Dominicana: hablan raro, tienen un cantao y usan mucho la $i$; hablan feo, dicen haiga, puieta, papei;

\footnotetext{
8 Tanto Lares en Puerto Rico como El Cibao en República Dominicana son sitios estigmatizados lingüísticamente en estos países por considerarse su habla rural y por la alta frecuencia de determinados fenómenos fonéticos, como la vocalización de la vibrante.
} 
- República Dominicana sobre Puerto Rico: tienen un tono diferente; arrastran la erre; hablan espanglish.

\subsection{Comportamiento de las variables sociolingüísticas}

En las variables sociolingüísticas estudiadas, específicamente en la variable sexo, las mujeres cubanas y puertorriqueñas destacan por sobre los hombres en la consideración de criterios lingüísticos para establecer las semejanzas y diferencias entre variedades $(36,5 \%$ sobre $30,6 \%$ las cubanas y $44,6 \%$ sobre $28,9 \%$ las boricuas en cuanto a semejanzas; $45,8 \%$ las mujeres frente a $42,1 \%$ los hombres en Cuba y $49,4 \%$ frente a $39 \%$ en Puerto Rico para las diferencias). En general, es el sexo femenino quien manifiesta una mayor conciencia lingüística al tomar en cuenta un número mayor de criterios en sus respuestas, especialmente las mujeres puertorriqueñas más jóvenes (IGE), pues son las únicas en considerar cuestiones relacionadas con la cercanía geográfica, la rapidez elocutiva, el uso del espanglish y la incorrección.

La variable edad no arrojó prácticamente resultados destacables. Por niveles de instrucción, de los datos se deduce que el desconocimiento es mayor mientras menor es el nivel educacional de los informantes, los de nivel bajo son los que más declaran no saber. Al mismo tiempo, a medida que aumenta la instrucción aumenta también la consideración de los niveles lingüísticos para establecer las semejanzas (léxico, fónico, suprasegmental). Esto sucede en Cuba y Puerto Rico; en Cuba especialmente los de nivel bajo no toman en cuenta en ninguna respuesta los niveles léxico y fónico.

\subsection{Análisis perceptual}

Si se analizan los mapas perceptuales de semejanzas elaborados a partir de las respuestas de cada país (Mapas 1, 2 y 3), se aprecia que los cubanos y puertorriqueños evidenciaron tener una percepción de un área lingüística similar en la que ubican las Antillas, Venezuela y, en menor medida, Colombia y Panamá. Sin embargo, los dominicanos mostraron una percepción mucho más amplia de su área lingüística en la que, además de estas variedades, incluyen las de México y España.

En cada uno de estos mapas mentales resulta evidente que se cumple el principio de proximidad propuesto por Montgomery (2012), fundamentalmente el principio asociado a la proximidad geográfica, según el cual lo más cercano es lo mayormente considerado por los hablantes. Asimismo, se manifiesta la proximidad en cuanto a relevancia cultural, es por ello que variedades de países no tan próximos geográficamente están entre los más similares, como es el caso de España y México. En ello entran en juego factores de relevancia histórica, cultural y de prestigio; de España se ha reiterado el prestigio histórico que le conceden los hablantes antillanos (Alvar 1986; Mojica 2014; Severino 2014; Sobrino et al. 2014) y con México influyen, además, las estrechas relaciones culturales y la influencia de los medios de comunicación, fundamentalmente la televisión. 
A partir de la información obtenida se han elaborado mapas perceptuales generales de semejanzas y diferencias para ofrecer una visión global de las percepciones dialectales de los hispanohablantes antillanos. En ellos se representan solo aquellas variedades nacionales que coinciden en ser mencionadas por cubanos, puertorriqueños y dominicanos con frecuencias por encima de la media. El mapa perceptual general de semejanzas (Mapa 7) ilustra que el área dialectal coincidente en los mapas mentales de los antillanos es la que comprende los tres países y Venezuela.

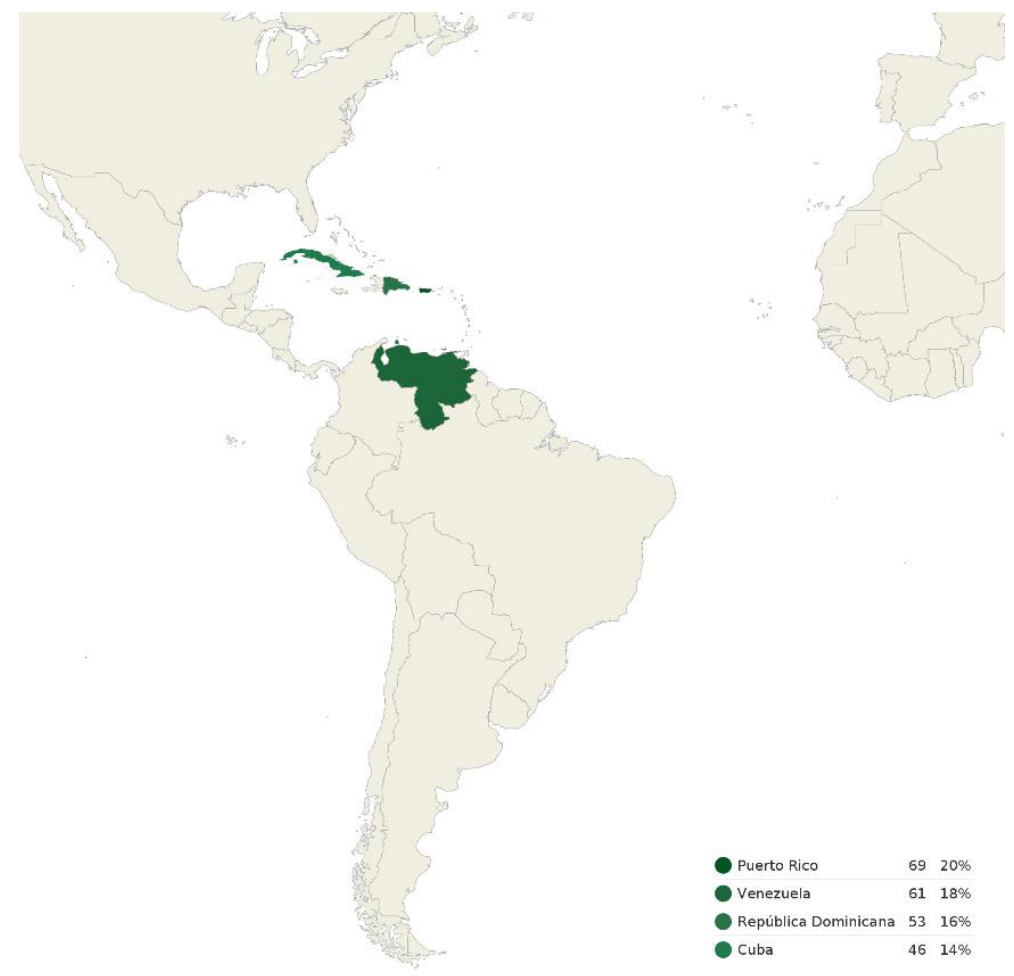

Mapa 7: Mapa perceptual general de variedades consideradas semejantes

Esta área dialectal no estaría muy alejada de las divisiones dialectales aportadas desde la dialectología tradicional (Zamora y Guitart 1988) y los argumentos expuestos por los hablantes comunes han sido también validados por especialistas al reconocer las similitudes existentes (Tejera 1999, 187).

Sobre las percepciones acerca de las diferencias entre variedades, en los tres países se mencionaron algunas distantes geográficamente junto a otras que coinciden con las consideradas semejantes (Mapas 4, 5 y 6). Los cubanos incluyen entre los países diferentes a ellos lingüísticamente a Venezuela y Colombia, por ejemplo; los puertorriqueños mencionan Colombia y República Dominicana; mientras que los dominicanos en su mapa perceptual de diferencias calcan prácticamente lo concebido como semejante y solo añaden Chile y Argentina. Por tanto, no se cumple claramente el principio relacionado con la existencia de un continuum dialectal perceptual puesto que no hay una correspondencia con el hecho de que a mayor distancia mayor diferenciación, sino que podría aplicarse también aquí el principio de proximidad: lo más conocido es lo que forma parte del mapa mental del hablante y es a la información que recurre para señalar tanto semejanzas como diferencias (Mapa 8). 


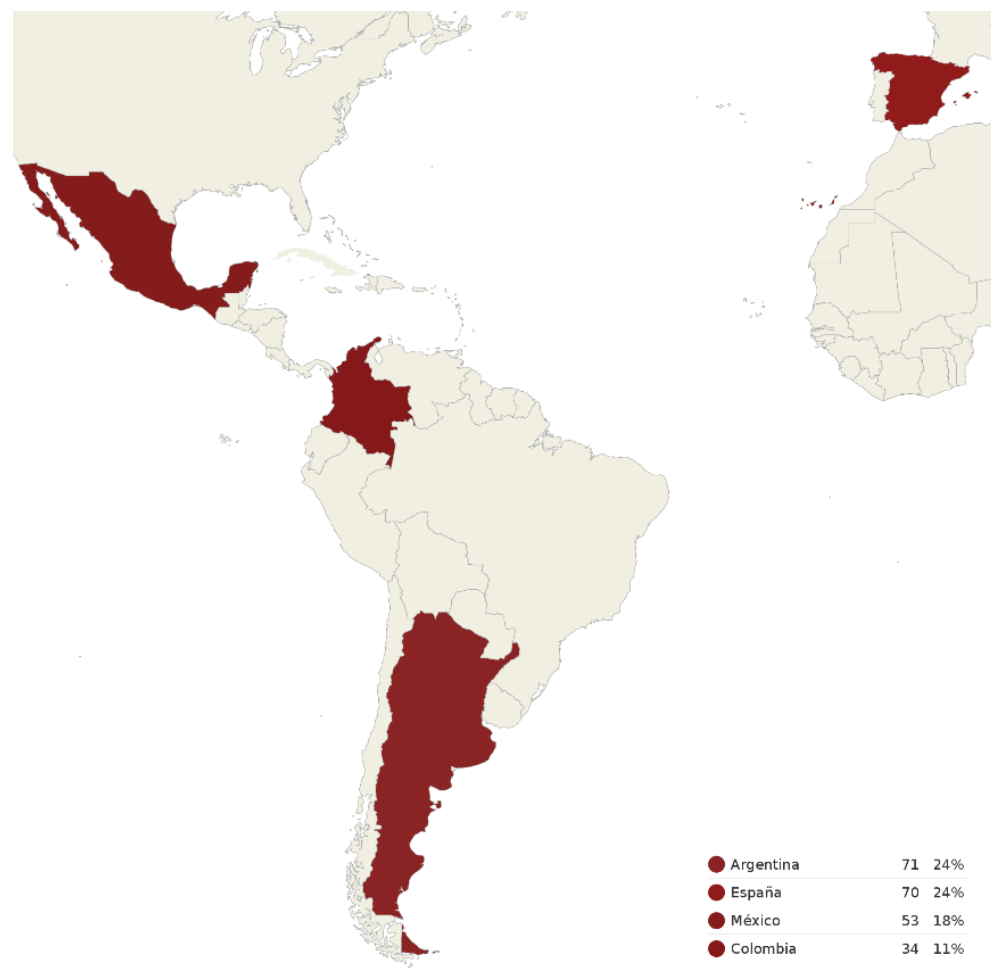

Mapa 8: Mapa perceptual general de variedades consideradas diferentes

En República Dominicana con frecuencia un informante consideró una variedad como semejante y diferente al mismo tiempo, esto sucedió con las variedades española, mexicana y puertorriqueña. En todos estos casos los argumentos para considerarlas semejantes se basaron en el empleo de una lengua común, criterio muy tomado en cuenta por los dominicanos, mientras las explicaciones de estas variedades como diferentes se enfocaron en la pronunciación, en el caso de la modalidad española, y en rasgos suprasegmentales (cantadito) y uso del espanglish de los puertorriqueños.

Si se representan las frecuencias relativas de semejanzas y diferencias por países, según los datos en conjunto de las tres capitales trabajadas (Gráfico 1), puede apreciarse que, a pesar de que casi todos los países fueron considerados tanto para semejanzas como para diferencias, la percepción en cuanto a similitudes de los antillanos se concentra en las Antillas y Venezuela; Panamá y Colombia estarían ubicadas en la zona limítrofe del área dialectal que los antillanos conciben en sus mapas mentales, de ahí que estén muy equilibradas las percepciones de semejanzas y diferencias que se manifiestan hacia la variante colombiana. 


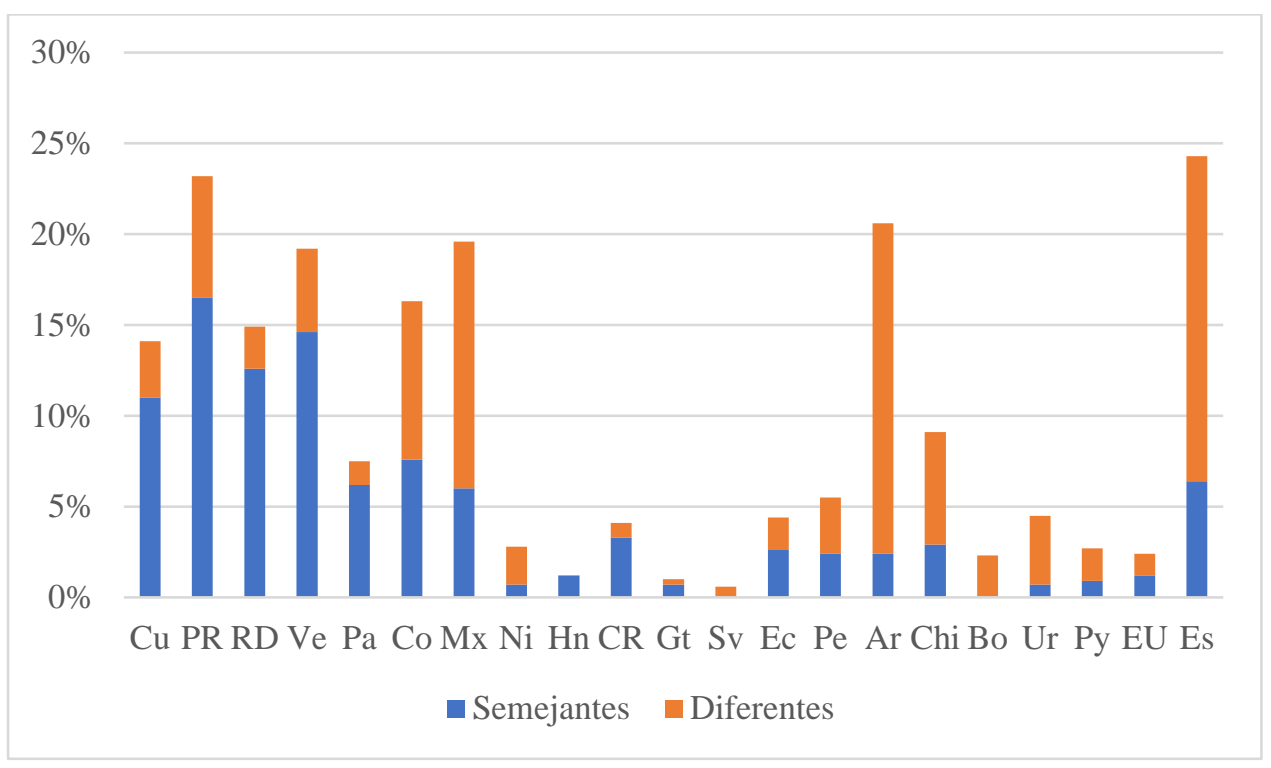

Gráfico 1: Relación de percepción de semejanzas y diferencias por países

La zona centroamericana es la menos mencionada: a excepción de Panamá, países como Honduras, Guatemala y El Salvador se presentan como los grandes desconocidos, hacia estas variantes prácticamente no se dirige ninguna opinión. El Sur, si bien no destaca como una región de la que se tenga gran dominio, se presenta como un área percibida como diferente lingüísticamente en la que sobresalen las variedades argentina y chilena. Además de estos países suramericanos, destacan México en el norte y también España como focos hacia los que predomina una perspectiva diferenciadora.

La representación de estos datos en una gráfica de escala multidimensional ${ }^{9}$ permite visualizar la distribución espacial de las variedades según las percepciones dialectales de los antillanos. En el Gráfico 2 se confirma que los antillanos perciben un gran grupo de variedades de lengua española donde se ubican la mayoría de las variantes centro y suramericanas y otro conjunto, algo distante del anterior, donde incluyen sus variedades, todas ellas próximas entre sí: la cubana, dominicana, venezolana y puertorriqueña. Alejadas de estos dos subconjuntos, y con considerable distancia entre ellas, se encontrarían las variantes colombiana, mexicana, española y argentina.

\footnotetext{
9 El Escalamiento Multidimensional (Multidimensional Scaling, MDS) es una técnica multivariante que representa en un espacio geométrico las proximidades existentes entre un conjunto de objetos.
} 


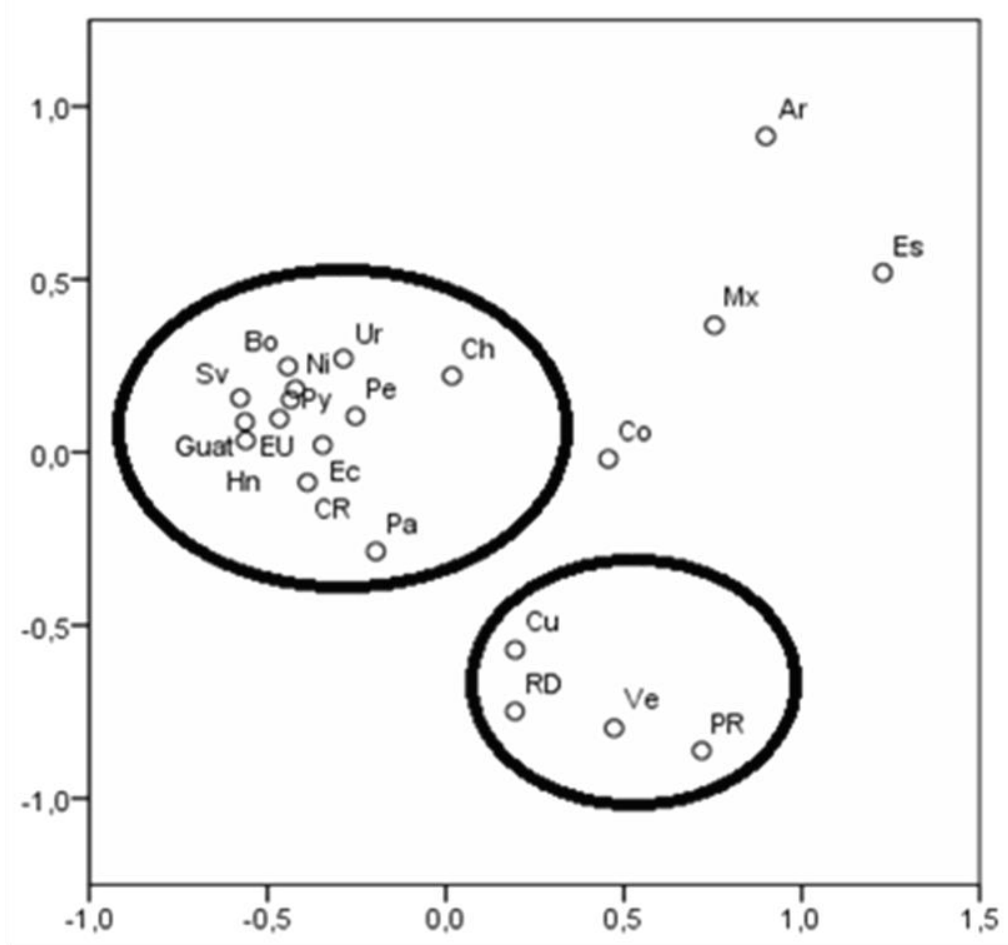

Gráfico 2: Escala multidimensional

En cuanto a las relaciones perceptuales existentes entre los antillanos puede afirmarse que, de modo general, se cumple el principio de la reciprocidad, esto si se toma en cuenta que cada una de las Antillas incluye en sus mapas perceptuales a las otras dos restantes. No obstante, lo que podría denominarse «la intensidad del parámetro de la reciprocidad» varía de un país a otro. Entre cubanos y puertorriqueños se cumpliría cabalmente el principio de la reciprocidad, en tanto los cubanos sienten su variante similar a la puertorriqueña y viceversa, con frecuencias de mención significativas y muy similares. Los vínculos con República Dominicana toman un matiz diferente ya que consideran los lazos con respecto a los cubanos mucho más débiles (10\% de semejanzas) que a la inversa, y entre dominicanos y boricuas la relación es más débil que entre boricuas y cubanos; puede entenderse que puertorriqueños y dominicanos consideran sus variantes medianamente semejantes.

\section{Conclusiones}

Las percepciones dialectales de los hispanohablantes de las Antillas evidencian que ellos circunscriben sus variedades a un área dialectal común caracterizada por rasgos lingüísticos similares, fundamentalmente en los niveles fonéticos, léxicos y suprasegmentales, así como por las semejanzas de rasgos culturales. Al mismo tiempo, esta zona dialectal perceptual estaría alejada de otras variedades como la argentina, española, mexicana y colombiana que se caracterizan como marcadamente diferenciadas de las propias y constituyen normas hispánicas regionales prestigiosas. Todo ello, sumado al matiz estigmatizador con que se presentan algunos rasgos al caracterizar las variedades consideradas similares, denota cierta auto discriminación lingüística hacia el área perceptual propia dentro del contexto panhispánico. 
Aunque, en general, las percepciones dialectales de cubanos, puertorriqueños y dominicanos coinciden en muchos de los aspectos tratados, pueden distinguirse algunas cuestiones entre unos y otros. Los cubanos, por ejemplo, manifiestan una percepción de unidad dialectal antillana mucho más fuerte que sus vecinos; los puertorriqueños, en un triángulo de relaciones, se ven mucho más vinculados a Cuba que a República Dominicana, y los dominicanos manifestarían una conciencia más débil de la vinculación existente entre las tres variedades. Los tipos de respuestas ofrecidas apoyan también esta idea. Los cubanos y puertorriqueños fueron más propensos a aportar respuestas monoperceptuales y biperceptuales en las que restringen los vínculos de su variedad a solo una o dos modalidades de español, generalmente vecinas. Por el contrario, los dominicanos concibieron respuestas multiperceptuales en las que reflejan que sus mapas perceptuales están constituidos por diversas variedades en las que no tienen predominio, necesariamente, las vecinas antillanas.

Los datos acopiados a partir de los postulados de la dialectología perceptual constituyen información esencial para un estudio sobre actitudes lingüísticas en las Antillas. Igualmente, el conocimiento de cómo se perciben a sí mismos como hablantes de español desde un punto de vista lingüístico y dialectológico los hablantes cubanos, puertorriqueños y dominicanos, y los vínculos que establecen entre ellos, dan luces sobre la identidad lingüística nacional y regional de la zona antillana.

\section{Bibliografía}

Alvar, Manuel. 1986. Hombre, etnia, estado. Actitudes lingüísticas en Hispanoamérica. Madrid: Editorial Gredos.

Bravo, Ana. 2015. "Mapas mentales y actitudes lingüísticas. El caso del murciano a través de los trabajos de campo de estudiantes del grado de Lengua y Literatura españolas de la Universidad de Murcia".

https://www.researchgate.net/publication/279877403_MAPAS_MENTALES_y_ACTI TUDES_LINGUISTICAS_EL_CASO_DEL_MURCIANO_A_TRAVES_DE_LOS_T RABAJOS_DE_CAMPO_DE_ESTUDIANTES_DEL_GRADO_DE_LENGUA_Y_L ITERATURA_ESPANOLAS_DE_LA_UNIVERSIDAD_DE_MURCIA

Castro, Thais y Irania Malaver. 2016. "División dialectal del español venezolano: estudio dialectológico perceptivo". Dialectología (16): 19-43.

Chiquito, Ana Beatriz y Miguel Ángel Quesada Pacheco (eds.). 2014. Actitudes lingüísticas de los hispanohablantes hacia el idioma español y sus variantes, Vol. 5. Bergen Language and Linguistic Studies (BeLLS)

https://bells.uib.no/bells/issue/current/showToc.

Choy López, Luis Roberto. 1999. Periodización y orígenes en la historia del español de Cuba. Universitat de Valencia: Tirant lo Blanch Libros.

Erdözová, Zuzana. 2011. "El español de México en los ojos de sus hablantes. Un estudio desde la sociolingüística y la dialectología perceptiva”. Lengua y voz 1(1): 57-81.

Fúster, Nadja. 2012. "Percepciones y actitudes lingüísticas de habaneros y santiagueros en Cuba". Tesis de maestría, Universidad de Puerto Rico, Recinto Universitario de Río Piedras. 
Lipski, John. 2004. El español de América. Madrid: Cátedra.

Long, Daniel y Dennis Preston. 2002. Handbook of perceptual dialectology (Vol. 2). Amsterdam: John Benjamins Publishing.

Preston, Dennis. 1999a. Handbook of perceptual dialectology (Vol. 1). Amsterdam: John Benjamins Publishing.

. 1999b. "A language Attitude Analysis of Regional US Speech: Is Northern US English Not Friendly Enough?” Cuadernos de Filología Inglesa 8: 129-146.

. 2004. "Language with an Attitude". En The Handbook of Language Variation and Change, editado por Jack Chambers, Peter Trudgill y Natalie Shilling-Estes, 40-66. Oxford: Blackwell.

Quesada Pacheco, Miguel Ángel. 2013. "División dialectal de Costa Rica según sus hablantes". Dialectologia et Geolinguistica (21): 36-69.

. 2014. "División dialectal del español de América según sus hablantes. Análisis dialectológico perceptual". Boletín de Filología, XLIX (2): 257-309.

Mojica De León, Carla M. 2014. "Una mirada hacia las actitudes lingüísticas en Puerto Rico". En Actitudes lingüísticas de los hispanohablantes hacia el idioma español y sus variantes, editado por Ana Beatriz Chiquito y Miguel Ángel Quesada, Vol. 5, 12491315), Bergen Language and Linguistics Studies (BeLLS). https://bells.uib.no/bells/article/view/693.

Montgomery, Chris. 2012. "The effect of proximity in perceptual dialectology". Journal of Sociolinguistics 16(5): 638-668.

Moreno Fernández, Francisco. 2015. "La percepción global de la similitud entre variedades de la lengua española". En Les variations diasystematiques et leurs interdependances dans les langues romanes, editado por Kirsten Jeppesen Kragh y Jan Lindschouw, 217-238. Strasbourg.

Moreno Fernández, Juliana y Francisco Moreno Fernández. 2002. "Madrid Perceptions of Regional Varieties in Spain". En Handbook of Perceptual Dialectology, editado por Daniel Long y Dennis Preston, Vol. 2, 295-320. Philadelphia: John Benjamins.

Serrano, Julio César. 2002. “Cuántos dialectos del español existen en México? Ensayo de dialectología perceptual". Manuscrito.

http://lef.colmex.mx/Sociolinguistica/Cambio\%20y\%20variacion/Ensayo\%20de\%20di alectologia\%20perceptual.pdf.

Severino, Glennys. 2014. "Actitudes lingüísticas en República Dominicana. Conciencia e identidad lingüísticas en la ciudad de Santo Domingo". En Actitudes lingüísticas de los hispanohablantes hacia el idioma español y sus variantes, editado por Ana Beatriz Chiquito y Miguel Ángel Quesada, Vol. 5, 1316-1345, Bergen Language and Linguistics Studies (BeLLS). https://bells.uib.no/bells/article/view/694.

Sobrino Triana, Roxana. 2013. "El español en Cuba según sus hablantes: un acercamiento desde la dialectología perceptiva”. En Memorias Lingüística 2013 (CD-Rom), editado por Instituto de Literatura y Lingüística "José Antonio Portuondo Valdor". La Habana. . 2017. "Actitudes lingüísticas en el Caribe insular hispánico". Tesis doctoral, Universidad de Bergen.

Sobrino Triana, Roxana, Lourdes Montero y América Menéndez. 2014. "Actitudes lingüísticas en Cuba. Cambios positivos hacia la variante nacional de lengua". En 
Actitudes lingüísticas de los hispanohablantes hacia el idioma español y sus variantes, editado por Ana Beatriz Chiquito y Miguel Ángel Quesada, Vol. 5, 290-408, Bergen Language and Linguistics Studies (BeLLS). https://bells.uib.no/bells/article/view/682

Tejera, María J. 1999. “Orígenes y causas históricas de la homogeneidad del habla del caribe hispánico y de la pluralidad de normas. Las comunicaciones oficiales y los vínculos informales". En Identidad cultural y lingüística en Colombia, Venezuela y en el Caribe hispánico, editado por Matthias Perl y Klaus Pörtl, 187-195. Tübingen: Niemeyer.

Valdés, Sergio. 1999. “Transculturación e identidad lingüística en el Caribe hispánico”. En Identidad cultural y lingüística en Colombia, Venezuela y en el Caribe hispánico, editado por Matthias Perl y Klaus Pörtl, 241-248. Tübingen: Niemeyer.

Zamora Munné, Juan C. y Jorge M. Guitart. 1988. Dialectología hispanoamericana. Teoríadescripción-historia. Salamanca: Ediciones Almar. 